\title{
Circular frames of the humerus: salvage surgery case series
}

\author{
Pretorius $\mathrm{HS}^{1} \mathbb{1}^{\mathrm{D}}$, Strauss $\mathrm{K}^{2}$, Ferreira $\mathrm{N}^{3} \mathbb{0}$, Lamberts $\mathrm{RP}^{4}$ \\ 1 BSc, MBChB, MMed(Orth)(Stell); Senior Specialist* \\ 2 MBChB, FCOrth, MMed(Orth)(SA); Registrar* \\ 3 BSc, MBChB, FCOrth, MMed(Orth)(SA); Associate Professor* \\ 4 MSc (Med HMS), PhD (Exer Physiol), FECSS; Professor and Head of Research* \\ * Division of Orthopaedic Surgery, Department of Surgical Sciences, Faculty of Medicine and Health Sciences, Stellenbosch University, Tygerberg, \\ South Africa
}

Corresponding author: Dr HS Pretorius, Division of Orthopaedic Surgery, Department of Surgical Sciences, Faculty of Medicine and Health Sciences, Stellenbosch University, Tygerberg Campus, Tygerberg, South Africa; PO Box 19063, Tygerberg, 7505; tel +27 21938 9266; email: hsp359@sun.ac.za

\begin{abstract}
Introduction: High energy fractures like gunshot-related injuries or high velocity road traffic accidents are often complex to treat and have been associated with a higher non-union rate, especially when there is extensive bone loss. Fractures with severe comminution may not heal due to bone loss and an inability to achieve adequate fracture stability. Treatment of fractures that are not amenable to plating or nailing may lead to non-unions, septic or aseptic, that need repeat surgeries. Stabilisation with external fixation is a reliable option to maintain stability and provide a suitable environment for union.

Materials and methods: This retrospective study used medical records of all patients with complex mid-shaft humerus fractures, as well as their complications, that were treated with hexapod circular external fixation between January 2009 and September 2015. All the patients in this case series presented with severe humerus fractures or complications thereof which were not amenable to conventional therapy.

Results: Union was achieved in ten out of the 12 cases (83.3\%). Union was achieved without bone graft or any other interventions at the fracture site except implant removal where indicated. The median time in external fixation was 196 days (interquartile range: 112-228). The most common complication encountered in this series was pin-site infection. Fixation points were noted to be infected in $33 \%$ of cases at some time during the treatment period.

Conclusion: This study suggests that humeral non-unions and complex humerus fractures that are not amenable to conventional fixation methods, such as intramedullary nails and plates, can successfully achieve union when treated with a hexapod circular external fixator.
\end{abstract}

Level of evidence: Level 4

Keywords: Taylor spatial frame, complex humerus fractures, non-union, septic non-unions

Citation: Pretorius HS, Strauss K, Ferreira N, Lamberts RP. Circular frames of the humerus: salvage surgery case series. SA Orthop J 2019;18(4):3540. http://dx.doi.org/10.17159/2309-8309/2019/v18n4a4

Editor: Dr F Birkholtz, University of Pretoria, Pretoria, South Africa

Received: March 2019

Accepted: June 2019

Published: November 2019

Copyright: @ 2019 Pretorius HS. This is an open-access article distributed under the terms of the Creative Commons Attribution Licence, which permits unrestricted use, distribution and reproduction in any medium, provided the original author and source are credited.

Funding: The authors declare that they have no conflict of interest.

Conflict of interest: No benefits in any form have been received or will be received from a commercial party related directly or indirectly to the subject of this article. 


\section{Introduction}

High energy fractures like gunshot-related injuries (Figure 1) or high velocity motor vehicle accidents (MVAs) are often complex to treat and have been associated with a higher non-union rate, especially when there is extensive bone loss. ${ }^{1}$ Conventional management of these fractures would include an intramedullary nail or plate fixation to span the fracture while maintaining stability. Fractures with severe comminution may not heal due to bone loss and an inability to achieve adequate fracture stability. When the fixation fails due to non-union or infection, the use of external fixation is often required and may even be required as the initial fracture management due to severe soft tissue defects. ${ }^{1-3}$ In 2007, Tomic used llizarov circular external fixation to treat 28 non-unions of the humerus and achieved $100 \%$ union in all 28 cases in an average period of 4.1 months (range: $3.5-5.7$ months). ${ }^{4}$ In 2016, Ferreira et al. used circular external fixation to treat septic non-unions in eight cases and achieved $100 \%$ union rates with a mean fixator time of 29 weeks. ${ }^{5}$ A vital procedural step performed in this study was the radical debridement of devascularised bone ends and excision of the interposed fibrous tissue, which meant exposing the fracture site.

The purpose of this study was to present our experience with the use of hexapod circular external fixation of the humerus for a variety of indications.

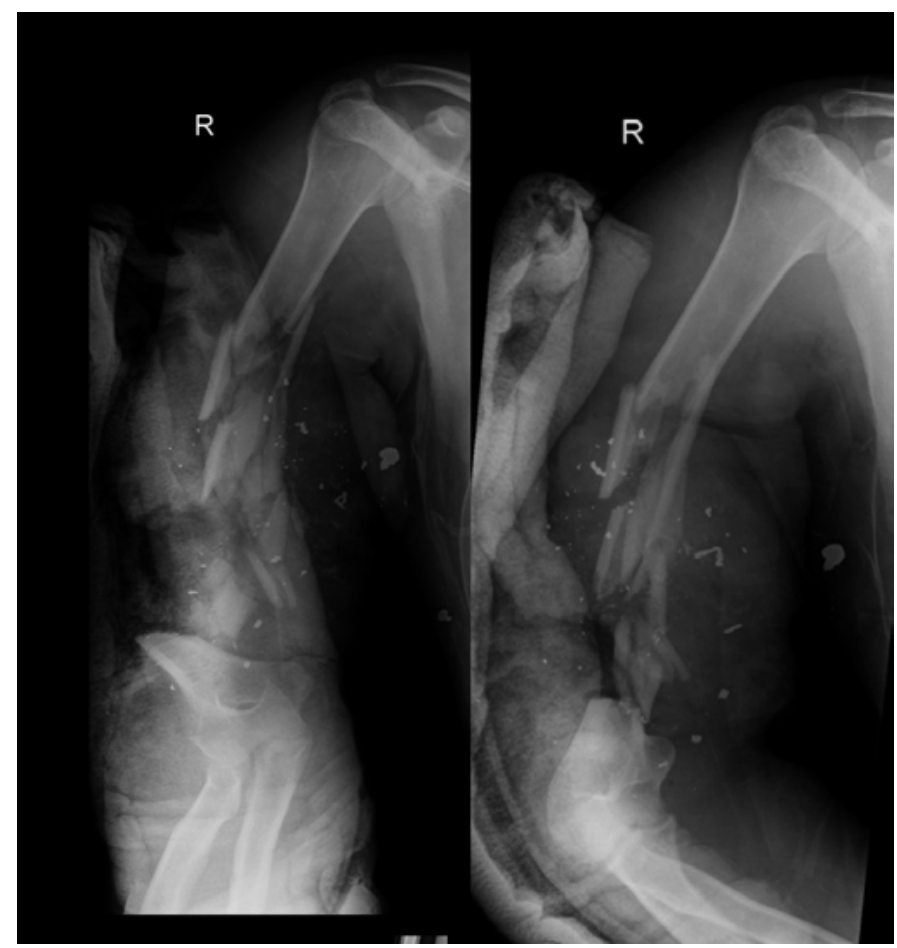

Figure 1. X-ray to illustrate the comminuted humerus fracture due to a gunshot wound that confirms the difficulty of conventional management

\section{Materials and methods}

This retrospective study used medical records of all patients with complex mid-shaft humerus fractures, as well as their complications, that were treated with hexapod circular external fixation between January 2009 and September 2015. All the patients in this case series presented with a severe humerus fracture or a complication thereof which was not amenable to convention therapy. An external fixator was the only viable option for management. All patients between the ages of 18 and 75 years were eligible for inclusion.

All patients were treated by the method of circular external fixator application to the humerus, using the Taylor spatial frame
(TSF) (Smith \& Nephew, Memphis, TN). Some cases required the removal of metal prior to fixator application, and intra-operative tissue samples were taken for microscopy, culture and sensitivity via the same approach used for the index procedures in the case of plate removal. No bone grafting was done for any of the fractures. Suppressive antibiotics were given in all septic cases.

A single stack TSF (5/8th ring proximal, and 5/8th ring distal) with six variable length struts was the standard configuration used. Rings were fixed with the open side of the proximal ring facing medial and the open side of the distal ring facing dorsal to allow elbow movement (Figures 2 and 3).

Fixation points varied according to the regional anatomy and wounds, but a minimum of two hydroxyapatite (HA)-coated half pins were inserted in the proximal segment. Distal fixation consisted of two to three fixation points as a combination of fine wires and HA pins. All fixation points adhered to described safe zones. ${ }^{6}$ Meticulous attention to the orthogonal mounting of the fixator as well as the correct pin insertion technique, with the avoidance of thermal necrosis by constant cooling with water while drilling, was performed as standard practice. After frame application the fracture was aligned as well possible in the coronal and sagittal planes under fluoroscopy, after which the fast-fix struts were locked.

Pin sites were cleaned, and gauze swabs impregnated with chlorhexidine and alcohol were applied. All patients received three doses of post-operative antibiotic prophylaxis and pin-site care was commenced on day 3 post-operatively in the ward. Wound dressings were exchanged for clean ones on day 3 and radiographs were obtained to determine the quality of the reduction. Patients who required adjustments were kept as inpatients until completion of the reduction. Patients were taught how to perform pin-site care by trained ward staff, and materials for this (chlorhexidine and gauze swabs) were dispensed on discharge. The first follow-up appointment was booked at the outpatient department no later than two weeks from the surgery date.

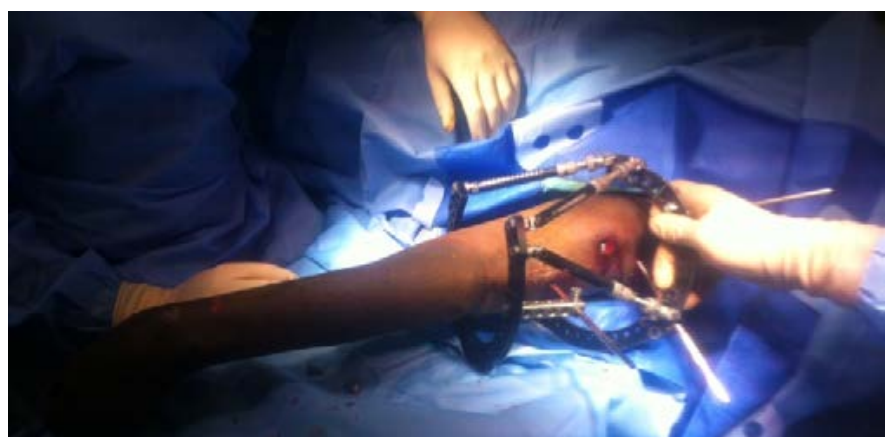

Figure 2. Intra-operative photograph taken after circular external fixator application, demonstrating full extension without tethering of soft tissues

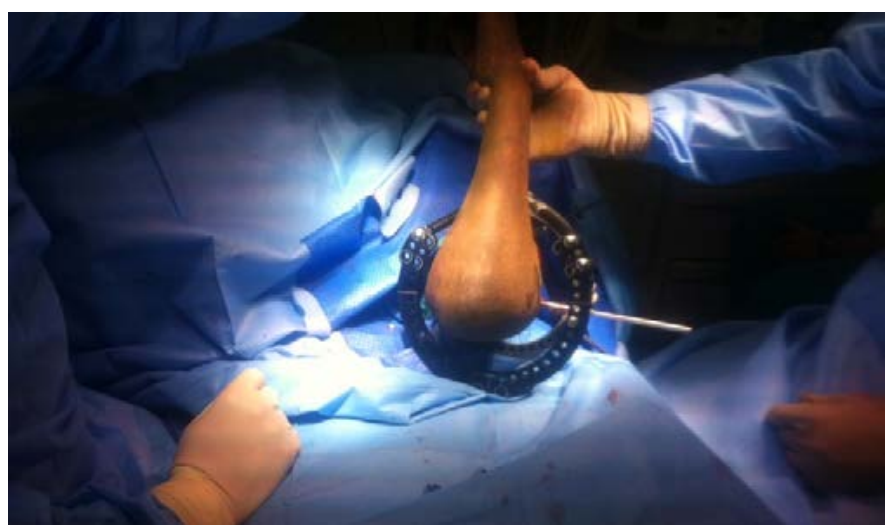

Figure 3. Intra-operative photograph of the same patient as in Figures 1 and 2 , illustrating correct frame positioning and full flexion possible 
Table I: Summary of characteristics of each patient in the series

\begin{tabular}{|c|c|c|c|c|c|}
\hline Patient & Fracture & $\begin{array}{l}\text { Mechanism of } \\
\text { injury* }\end{array}$ & $\begin{array}{l}\text { Previous } \\
\text { implants }\end{array}$ & Specific treatment & Aetiology \\
\hline 1 & Open & MVA & Nail & Implant removed and fracture not opened or biopsy done & Septic non-union \\
\hline 2 & Open & MVA & None & & Trauma \\
\hline 3 & Open & Assault & Nail & Implant removed and fracture not opened or biopsy done & Atrophic non-union \\
\hline 4 & Closed & FOOSH & None & & Atrophic non-union \\
\hline 5 & Closed & FOOSH & Nail & Implant removed and fracture not opened or biopsy done & Septic non-union \\
\hline 6 & Open & GSW & None & & Trauma \\
\hline 7 & Open & GSW & None & & Trauma \\
\hline 8 & Open & GSW & None & & Trauma \\
\hline 9 & Open & MVA & Nail & Implant removed and fracture not opened or biopsy done & Septic non-union \\
\hline 10 & Closed & MVA & Plate & Implant removed and fracture opened and biopsy done & Atrophic non-union \\
\hline 11 & Open & MVA & Nail & Implant removed and fracture not opened or biopsy done & Septic non-union \\
\hline 12 & Open & FOOSH & Plate & Implant removed and fracture opened and biopsy done & Atrophic non-union \\
\hline
\end{tabular}

*MVA: motor vehicle accident; GSW: gunshot wound; FOOSH: fallen on outstretched hand

Table II: Summary of injury characteristics, previous treatments and aetiology of each patient in the series

\begin{tabular}{|c|c|c|c|c|}
\hline Patient & Fracture & Mechanism of injury* & Previous implants & Aetiology \\
\hline 1 & Open & MVA & Nail & Septic non-union \\
\hline 2 & Open & MVA & None & Trauma \\
\hline 3 & Open & Assault & Nail & Atrophic non-union \\
\hline 4 & Closed & FOOSH & None & Atrophic non-union \\
\hline 5 & Closed & FOOSH & Nail & Septic non-union \\
\hline 6 & Open & GSW & None & Trauma \\
\hline 7 & Open & GSW & None & Trauma \\
\hline 8 & Open & GSW & None & Trauma \\
\hline 9 & Open & MVA & Nail & Septic non-union \\
\hline 10 & Closed & MVA & Plate & Atrophic non-union \\
\hline 11 & Open & MVA & Nail & Septic non-union \\
\hline 12 & Open & FOOSH & Plate & Atrophic non-union \\
\hline
\end{tabular}

*MVA: motor vehicle accident; GSW: gunshot wound; FOOSH: fallen on outstretched hand

\section{Radiological assessment}

Standard radiographs consisted of two orthogonal views, namely antero-posterior and lateral views, at each follow-up consultation. Additional radiographs were performed in select cases where residual deformity required further corrections. These consisted of radiographs centred on the specified reference ring.

Prior to removal of the fixator, the radiographs were scrutinised to assess union. In two cases the plain films were ambiguous in demonstrating union, and computerised tomography (CT) scans were performed in order to assess union. Two weeks following frame removal, all patients were followed up at the clinic where radiographs would be repeated and assessed for residual deformity.

\section{Functional assessment}

A functional assessment in the form of a Disabilities of the Arm, Shoulder and Hand (DASH) score was performed on all patients as part of their first follow-up visit following frame removal.

\section{Statistical analysis}

All data was captured from the patient medical records and entered into a Microsoft Excel spreadsheet. Data analyses were performed with Statistica 12.0 (Statsoft Inc. Tulsa, OK, USA). Due to the lack of homogeneity, descriptive statistics are expressed as median and interquartile ranges. Due to the descriptive nature of this case series, no inferential statistical analyses could be performed.

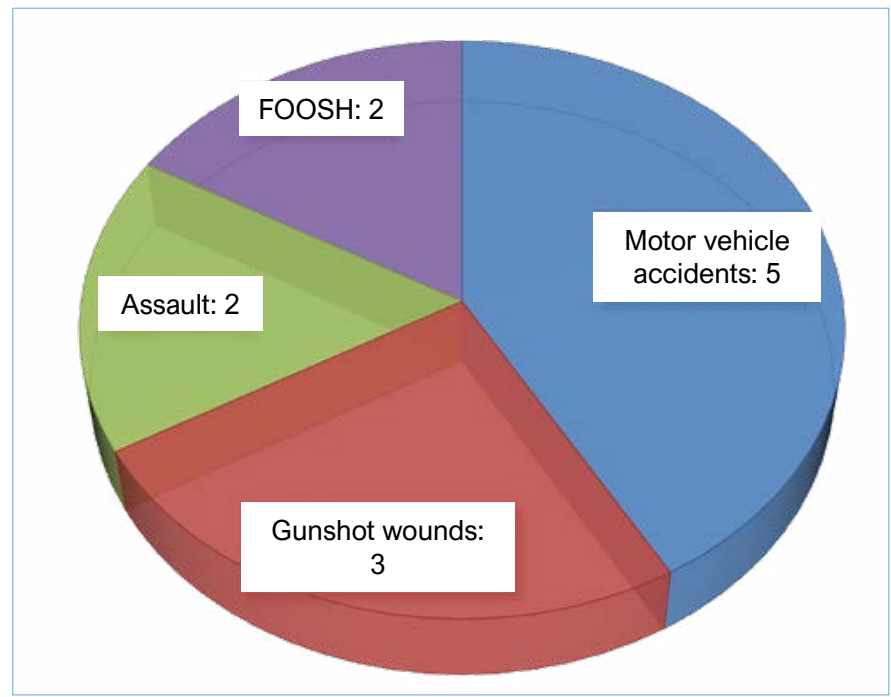

Figure 4. An overview of the mechanism of injury which caused the complex humerus fracture 


\section{Results}

The final cohort consisted of 12 patients with a median age of 53 years (interquartile range or IQR: 44-59). All the patients were right-hand dominant, and eight of the 12 had sustained a rightsided humerus fracture while the remaining four had a left-sided humerus fracture (Table I). The mechanism of injuries for the patient varied from MVAs and gunshot wounds to mechanical falls and assaults (Figure 4).

Nine fractures were open injuries while the remaining three were closed (Table II). Seven of the $12(58 \%)$ cases had previously been treated with internal fixation; of these, five patients had intramedullary nails while the remaining two had plates and screws. A total of four acute fractures and eight non-unions were treated in this series.

Union was achieved in ten out of the 12 cases (83.3\%). Union was achieved without bone graft or any other interventions at the fracture site except implant removal where indicated, and only in the plate cases were the fractures opened. The median time in external fixation was 196 days (IQR: 112-228). The overall limb length showed a maximum shortening of $4 \mathrm{~mm}$ (IQR: 1-3) in the operated humerus and a mean varus angulation of $3^{\circ}$ (IQR: 2-9). The bone contact and alignment were achieved intra-operatively with the frame (Figure 5).

Two cases failed to unite (Figures 6 and 7). In both of these cases the patients requested frame removal prior to completion of treatment. Against recommendations, the frames were removed. Custom-made thermoplastic splints were provided for these

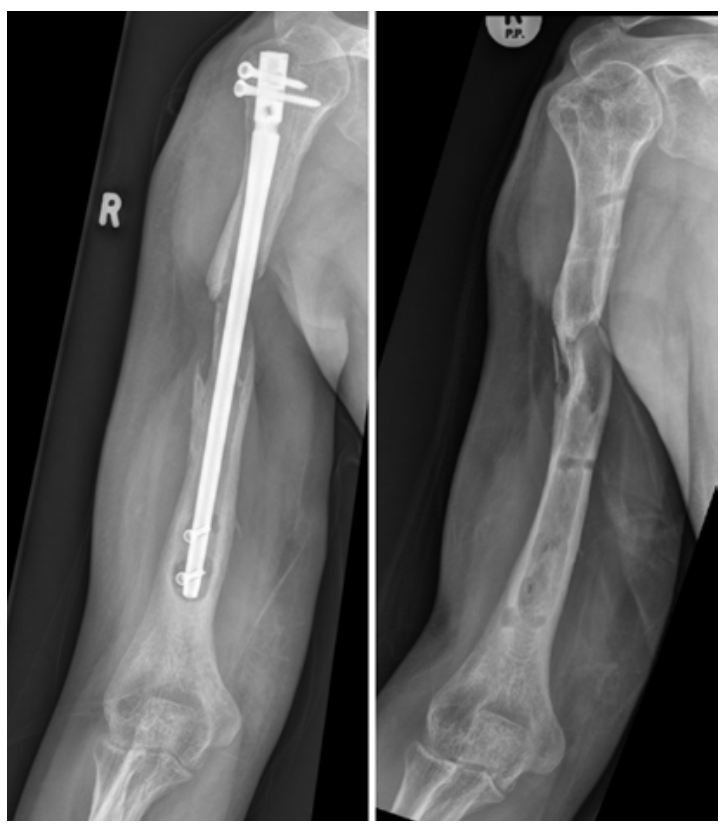

Figure 5. The X-ray on the left shows previous instrumentation in-situ, with evidence of sepsis and peri-implant osteolysis, in keeping with a diagnosis of septic non-union. The nail was removed and the fracture was not opened. The X-ray on the right of the same patient after circular external fixator had been removed, showing union patients and chronic suppressive antibiotic therapy was prescribed for the one case that had chronic osteomyelitis. Both cases had been referred to internal medicine to assist with the optimisation of the comorbidities at the outset of treatment, and appropriate medical therapies were instituted. In retrospect, both these cases probably represent failure on our part in terms of patient selection with regard to their comorbidities (Table III).

The median DASH scores at the first follow-up visit following fixator removal, was 70 (IQR: 41-76). According to the DASH score Work Module, seven of the 12 patients were employed prior to circular fixator treatment, three worked as manual labourers, while the remaining four performed sedentary work not requiring physical tasks. Three of these seven patients $(42.8 \%)$ returned to sedentary work within six weeks from the date of fixator application. Of the remaining four patients, a further two returned to work within three months. One patient changed occupation at the completion of treatment as he lost his job due to prolonged absence. One patient was unable to return to work in his former capacity.

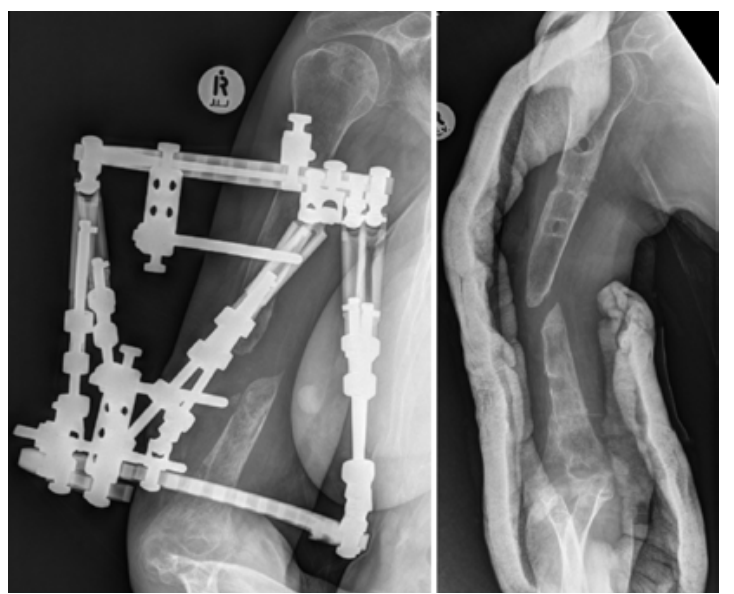

Figure 6. The X-ray on the left shows the frame collapse and failure due to loose pins. The X-ray on the right of the same patient shortly after frame removal, with U-slab applied. Note the erosive bone loss at each of the fixation points.

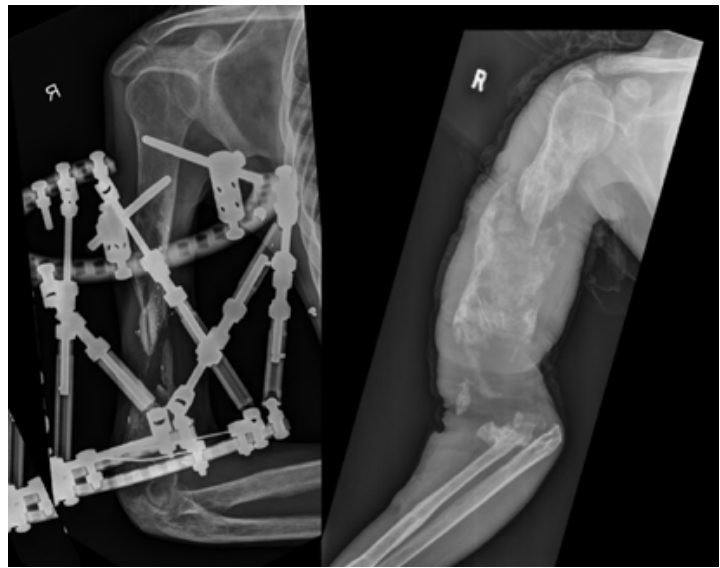

Figure 7. X-rays show the stabilised fracture in a reasonable position in a frame and severe bone destruction and fracture non-union after frame removal. Note the complete loss of the distal fragment.

Table III. Summary of patient characteristics that may have contributed to a poor outcome in failed cases

\begin{tabular}{|l|l|l|l|l|l|l|}
\hline Age (years) & \multicolumn{1}{|c|}{ Site } & Previous implants & \multicolumn{1}{|c|}{ Aetiology } & \multicolumn{1}{|c|}{ Risk factors } & Duration in frame (days) & Outcome \\
\hline 58 & Right humerus & None & Atrophic non-union & $\begin{array}{l}\text { Smoking, EtOH, } \\
\text { rheumatoid } \\
\text { arthritis, diabetes }\end{array}$ & 200 \\
\hline 50 & Left humerus & None & $\begin{array}{l}\text { Septic non-union } \\
\text { (trauma) }\end{array}$ & $\begin{array}{l}\text { Smoking, HIV+ve } \\
\text { (CD4 <350) }\end{array}$ & 184 \\
\hline
\end{tabular}


Regarding the Sports/Performing Arts module of the DASH score, there were four sportsmen among this group. Three had returned to their respective sporting activities and one had given up all sporting activities at the completion of treatment.

The most common complication that was encountered in this series was pin-site infection. Fixation points were noted to be infected in 33\% at some time during the treatment period (a total 20 out of 59 fixation points used). The majority of these complications (16 out of $20,80 \%$ ) were encountered at the proximal pins while only four out of $20(20 \%)$ of the distal fixation points became infected. In a single case, a septic proximal pin site was identified and treated at the first follow-up visit following frame application, but among the rest of the group there were no reports of pin-site problems in the first two months.

Pin-site infections were graded using the Checketts and Otterburn classification, ${ }^{7}$ and found to be grade 2 or less in all but one case. This isolated case proved to be a significant challenge in management due to ongoing chronic osteomyelitis, and eventually resulted in a failure to unite. Local sepsis control was not successful in this case despite removal of the offending pin. All other episodes of pin-site infection were managed successfully with a short course of oral antibiotics where indicated, local pin-site care and patient education.

\section{Discussion}

The aim of this study was to present our experience with the treatment of complex high energy humerus fractures and their associated complications, managed with hexapod circular external fixation. Although this treatment modality is very seldom needed for humerus fractures, a small subgroup of injuries remains difficult to treat by conventional means.

In our case series a union rate of $83.3 \%$ was achieved. This is similar to the expected outcome of the gold standard in treating humeral non-unions, namely autogenous iliac crest bone grafting and plating, ${ }^{4,8}$ without the need to expose the fracture/non-union site as in the study by Tomic et al. ${ }^{4}$ Sadiq, in 2002 , reported a $91 \%$ union rate of aseptic non-unions with compression plating and bone grafts, which is comparable to union results. ${ }^{8}$ The union rate of $83.3 \%$ achieved in this series supports the findings of McFayden et al. who successfully treated two humeral non-unions with TSFs. ${ }^{9}$ The aim of the treatment in the septic cases was to provide stability for the fracture: nails were removed without opening the fracture; and in the cases with a plate, the plate was removed and a biopsy done.

The other aspect of this type of limb reconstruction is the length of the limb after the treatment has been completed. In our series we did not find it necessary to actively shorten the humerus in any of these cases, which results in a more favourable residual deformity.

The use of internal fixation for the treatment of septic non-unions has not been reported in recent literature. Several articles report external fixation as the preferred method of fixation with or without debridement and secondary bone grafting for aseptic non-unions. This is an obvious advantage which would favour the use of the circular external fixator over internal fixation in the presence of sepsis. ${ }^{5,10-13}$

The complications encountered in this study offered some key learning points. The pin-site infection rate in this study was $33 \%$, which is significantly higher than the $17.9 \%$ incidence of pin-site sepsis found by Ferreira et al. in their study involving tibias, ${ }^{12}$ but also lower than the $41.6 \%$ pin-site sepsis rate quoted by Al-Sayyad in his study of TSFs of the upper limb in a paediatric population. ${ }^{13}$ The majority $(80 \%)$ of the problematic pin sites were related to the proximal pins, presumably as they traverse a thicker soft-tissue envelope than the distal pins. In our series, all patients were righthand dominant, and right-sided humerus fractures were twice as common as on the left, which meant that $66 \%$ of these patients had to perform pin-site hygiene with their non-dominant hand. For these reasons, we would recommend teaching a family member to assist the patient with pin-site care as this may lower infection rates and possibly the need for premature removal of pins.

The case series comprised an inhomogeneous group of patients which makes the results difficult to interpret. The sample size of 12 cases would appear to fall short when compared to studies of a similar nature conducted on tibias; ${ }^{14}$ however, it must be remembered that the underlying event rate of complex humeral fractures and non-unions is significantly lower than for the same pathology seen in tibias. ${ }^{14}$ Witness to this fact is the sum total of 12 cases collected in a time span of six years. When compared to studies involving circular external fixators of the upper limbs, however, this study is the largest of its kind according to our knowledge.

The functional assessment of these cases is based on the DASH score. However, we interpret these scores with caution as they were recorded at the first follow-up appointment following frame removal. Unfortunately, no follow-up DASH scores were available for comparison, and even though they are likely to show improvement over time, this is recognised as a limitation of this study.

\section{Conclusions}

In conclusion, this study suggests that complex humerus fractures that are not amenable to conventional fixation methods such as intramedullary nails and plates, as well as those cases that have failed to unite using these methods, can successfully achieve union when treated with a hexapod circular external fixator. An overall success rate of $83 \%$ was found, without the need for bone grafting or the excision of any bone that would lead to significant shortening. All the treatment failures occurred in non-union cases. Despite a limited functional capacity of the affected limb, the majority of patients were able to return to their previous level of function regarding work and even sporting activities. This treatment modality can, however, have a long duration, and is not without complications.

\section{Ethics statement}

The author/s declare that this submission is in accordance with the principles laid down by the Responsible Research Publication Position Statements as developed at the 2nd World Conference on Research Integrity in Singapore, 2010. Prior to commencement of the study ethical approval was obtained from the following ethical review board: Stellenbosch University Health Research Ethics committee, S15/09/208).

\section{Declarations}

The authors declare authorship of this article and that they have followed sound scientific research practice. This research is original and does not transgress plagiarism policies.

\section{Author contributions}

HSP (primary surgeon, contributed to the original study concept, primary author) and NF (contributed to the final manuscript) are orthopaedic surgeons with extensive clinical expertise in using Taylor spatial frames. RPL (contributed to the original study concept, data collection and analysis, contributed to the final manuscript) is an expert in the field of conducting orthopaedic research. KS (contributed to data collection analysis and managed the database, collation and contributed to the final manuscript) was a registrar at the time of the study.

\section{ORCID}

HS Pretorius (D) http://orcid.org/0000-0002-7419-0885 N Ferreira (D) http://orcid.org/0000-0002-0567-3373 RP Lamberts (D) http://orcid.org/0000-0003-1112-2604 


\section{References}

1. Keller A. The management of gunshot fractures of the humerus. Injury, 1995;26(2):93-96.

2. Hsu TL, Chiu FY, Chen CM, Chen TH. Treatment of non-union of humeral shaft fracture with dynamic compression plate and cancellous bone graft. J Chin Med Assoc. 2005;68:73-76.

3. March JL, Mahoney D, Steinbronn D. External fixation of open humerus fractures. lowa Orthop J. 1999;19:35-42.

4. Tomić S, Bumbasirević M, Lesić A, Mitković M, Atkinson HD. lizarov frame fixation without bone graft for atrophic humeral shaft nonunion: 28 patients with a minimum 2-year follow up. $J$ Orthop Trauma, 2007;21(8):549-56.

5. Ferreira N, Charles L, Serfontein C. Two stage reconstruction of septic non-union of the humerus with the use of circular external fixation. Injury, 2016;47(8):1713-18.

6. Nayagam S. Safe corridors in external fixation: the lower leg (tibia, fibula, hindfoot and forefoot). Strategies in trauma and limb reconstruction. 2007;2(2-3):105-10.

7. Checketts RG, Otterburn M, MacEachern AG. Pin track infection: definition, incidence and prevention. Int $J$ Orthop Trauma. 1993;3:16-18.

8. Sadiq SA. Non-union of the humeral shaft treated by internal fixation. International Orthopaedics, 2002;26(4):214-16.

9. McFayden I AR. The Taylor spatial frame in trauma and limb reconstruction surgery: report of 100 cases. J Bone Jt Surg Br. 2006;88:158-64.

10. Mostafavi HR, Tornetta P. Open fractures of the humerus treated with external fixation. Clin Orthop Rel Res. 1997;337:187-97.

11. Struijs PA, Poolman RW, Bhandari M. Infected nonunion of the long bones. J Orthop Trauma. 2007;21(7):507-11.

12. Ferreira N, Marais LC. Management of tibial non-unions: Prospective evaluation of a comprehensive treatment algorithm. SA Orthop J. 2016;1:60-66.

13. Al-Sayyad MJ. Taylor spatial frame in the treatment of upper extremity conditions. J Pediatr Orthop. 2012;32:169-78.

14. Ferreira $\mathrm{N}$, Aldous $\mathrm{C}$. The pathogenesis of tibial non-union. $S A$ Orthop J. 2016;15(1):51-59. 